\title{
Prostate-specific antigen nadir after high-dose-rate brachytherapy predicts long-term survival outcomes in high-risk prostate cancer
}

\author{
Hideyasu Tsumura, MD!, Takefumi Satoh, MD!, Hiromichi Ishiyama, MD², Ken-ichi Tabata, MD!, Shouko Komori, MD², \\ Akane Sekiguchi, MD², Masaomi lkeda, MD!, Shinji Kurosaka, MD!, Tetsuo Fujita, MD!', Masashi Kitano, MD², \\ Kazushige Hayakawa, MD², Masatsugu Imamura, MD'
}

'Department of Urology, '2Department of Radiology and Radiation Oncology, Kitasato University School of Medicine, Sagamihara, Japan

\begin{abstract}
Purpose: To evaluate the prognostic value of prostate-specific antigen nadir (nPSA) after high-dose-rate (HDR) brachytherapy in clinically non-metastatic high-risk prostate cancer patients.

Material and methods: Data from 216 patients with high-risk or locally advanced prostate cancer who underwent HDR brachytherapy and external beam radiation therapy with long-term androgen deprivation therapy (ADT) between 2003 and 2008 were analyzed. The median prostate-specific antigen (PSA) level at diagnosis was $24 \mathrm{ng} / \mathrm{ml}$ (range: 3-338 ng/ml). The clinical stage was T1c-2a in 55 cases (26\%), T2b-2c in 48 (22\%), T3a in 75 (35\%), and T3b-4 in $38(17 \%)$. The mean dose to $90 \%$ of the planning target volume was $6.3 \mathrm{~Gy} /$ fraction of HDR brachytherapy. After 5 fractions, external beam radiation therapy with 10 fractions of 3 Gy was administered. All patients initially underwent neoadjuvant ADT for at least 6 months, and adjuvant ADT was continued for 36 months. The median follow-up was 7 years from the start of radiotherapy.

Results: The 7-year PSA relapse-free rate among patients with a post-radiotherapy nPSA level of $\leq 0.02 \mathrm{ng} / \mathrm{ml}$ was $94 \%$, compared with $23 \%$ for patients with higher nPSA values (HR = 28.57; 95\% CI: 12.04-66.66; $p<0.001)$. Multivariate analysis revealed that the nPSA value after radiotherapy was a significant independent predictor of biochemical failure, whereas pretreatment predictive values for worse biochemical control including higher level of initial PSA, Gleason score $\geq 8$, positive biopsy core rate $\geq 67 \%$, and T3b-T4, failed to reach independent predictor status. The 7-year cancer-specific survival rate among patients with a post-radiotherapy nPSA level of $\leq 0.02 \mathrm{ng} / \mathrm{ml}$ was $99 \%$, compared with $82 \%$ for patients with higher nPSA values (HR $=32.25 ; 95 \% \mathrm{CI}: 3.401-333.3 ; p=0.002)$.

Conclusions: A post-radiotherapy nPSA value of $\leq 0.02 \mathrm{ng} / \mathrm{ml}$ was associated with better long-term biochemical
\end{abstract} tumor control even if patients had pretreatment predictive values for worse control.

J Contemp Brachytherapy 2016; 8, 2: 95-103 DOI: $10.5114 / j c b .2016 .59686$

Key words: brachytherapy, high-dose-rate, prostate cancer, PSA nadir.

\section{Purpose}

High-dose-rate (HDR) brachytherapy has been accepted as a useful method to control localized and locally advanced prostate cancers $[1,2,3]$. The combination of HDR brachytherapy and external beam radiation therapy (EBRT) is expected to provide an effective approach for delivering radiation doses more safely and precisely compared to EBRT alone in high-risk and locally advanced prostate cancer, even if patients have extracapsular invasion and seminal vesicle invasion $[4,5,6,7]$. Hypofractionated radiotherapy may prove advantageous for treating prostate cancer because of the low $\alpha: \beta$ ratio [8]. In addition, the use of long-term androgen deprivation ther- apy (ADT; $\geq 2$ years) with radiotherapy has been shown to contribute to better overall survival or cancer-specific survival in men with locally advanced or localized unfavorable-risk prostate cancer compared to radiotherapy alone $[9,10,11]$. Thus, we have treated unfavorable-risk prostate cancer with HDR brachytherapy combined with hypofractionated EBRT and long-term ADT. Although several reports describe the efficacy and safety for HDR brachytherapy combined with EBRT or hormonal therapy, limited data are available regarding the predictive value for the clinical outcome in this combination therapy $[4,5,12,13,14,15]$.

In this study, we report outcomes for high-risk and locally advanced prostate cancer patients treated with com-
Address for correspondence: Hideyasu Tsumura, MD, Department of Urology, Kitasato University School of Medicine, Sagamihara, Japan, 1-15-1 Kitasato Minami-ku, Sagamihara, Kanagawa 252-0374, Japan, phone: +81 42778 9091, fax: +81 42778 9374, 凶e-mail: tsumura@med.kitasato-u.ac.jp
Received: 07.12 .2015

Accepted: 10.04 .2016

Published: 27.04.2016 
bination of HDR brachytherapy, EBRT, and long-term ADT with a median follow-up of 7 years. We investigated whether prostate-specific antigen nadir (nPSA) after HDR brachytherapy is associated with long-term biochemical tumor control. We also investigated whether the lowest prostate-specific antigen (PSA) level achieved during the first year after completing radiotherapy could become an early surrogate marker for long-term biochemical control.

\section{Material and methods}

A total of 223 consecutive patients with high-risk prostate cancer defined according to D'Amico criteria (clinical stage T2c, or PSA > $20 \mathrm{ng} / \mathrm{ml}$, or biopsy Gleason score 8-10) were treated using a combination of HDR brachytherapy and hypofractionated EBRT with long-term ADT between December 2003 and July 2008 [16]. Pretreatment evaluation included clinical history, physical examination, blood laboratory findings, pelvic computed tomography (CT), pelvic magnetic resonance imaging, and a bone scan. Patients with clinically positive lymph nodes or distant metastasis were excluded. All biopsy slides were reviewed by our institutional pathologists. A castrate testosterone level was defined as $\leq 50 \mathrm{ng} / \mathrm{dl}$. The institutional review board approved this retrospective study (B14-202).

\section{High-dose-rate brachytherapy and hormonal therapy}

Our treatment protocol for HDR brachytherapy and hormonal therapy in high-risk prostate cancer was previously reported and shown in Figure 1 [5,7]. Briefly, treatment of HDR brachytherapy was initiated using placement of a closed transperineal hollow needle under transrectal ultrasound guidance. Multiple 20- to $25-\mathrm{cm}$ long, closed-end, 15-G plastic hollow needles were inserted transperineally using a Syed-Neblett plastic template (Alpha-Omega Services, Bellflower, CA, USA). Routinely, 18 needles were implanted. Flexible cystoscopy was conducted to check that the urethra had not been penetrated by the implanted tubes. The needle tips were left within the urinary bladder, $1.5 \mathrm{~cm}$ above the sonographically or cystoscopically defined base of the prostate. After all of these procedures had been completed, the patient underwent CT to obtain scans at $5 \mathrm{~mm}$ intervals for CT-based planning. Contours of the planning target volume (PTV), urethra, and rectum were outlined according to transverse CT images. The PTV was defined as the prostate gland with or without proximal seminal vesicles, with an additional 3 to $5 \mathrm{~mm}$ margin all around. Reference points for the urethra were set on the center of the urethral catheter, and those for the rectal wall were set $5 \mathrm{~mm}$ behind the edge of the anterior rectal wall on transverse CT images with $10 \mathrm{~mm}$ intervals. Reference points for the PTV were automatically distributed on the surface of the PTV. Dose limitation of HDR brachytherapy was set as $10 \mathrm{~Gy} /$ fraction for urethral reference points and $4 \mathrm{~Gy} /$ fraction for rectal reference points, and we attempted to prescribe $7.5 \mathrm{~Gy} /$ fraction to reference points of the PTV (unless the dose limitation was violated) using inverse planning and geometric optimization. Because of urethral and rectal dose limitations, covering the periphery of the PTV with the prescribed dose was difficult in some patients. The mean dose to $90 \%$ of the PTV $\left(\mathrm{D}_{90}\right)$, the prostate volume receiving at least $6 \mathrm{~Gy}\left(\mathrm{~V}_{6}\right)$, and the prostate volume receiving at least $9 \mathrm{~Gy}\left(\mathrm{~V}_{9}\right)$ were $6.3 \pm 0.6 \mathrm{~Gy}, 91 \% \pm 5 \%$, and $53 \% \pm 9 \%$ per fraction, respectively. Five fractions of HDR treatment were administered. After CT-based planning using a Nucletron planning system (Nucletron, an Elekta company, Elekta AB, Stockholm, Sweden), the first treatment session of HDR brachytherapy was conducted using the Nucletron microSelectron HDR ${ }^{192}$ Ir remote afterloading system (Nucletron, an Elekta company, Elekta $\mathrm{AB}$, Stockholm, Sweden). Dwell positions were activated at $5 \mathrm{~mm}$ intervals along each catheter. The deepest dwell position could be set at $6.5 \mathrm{~mm}$ from the catheter tip if needed. Catheter positions were checked by fluoroscopy before every treatment session and corrected if interfraction needle movements $>5 \mathrm{~mm}$ were noted. The first treatment session was conducted on the day of implantation, with the subsequent four treatment sessions administered twice daily with an interval of at least 6 hours between fractions. Treatment duration was thus 3 days. At $6^{\text {th }}$ day after completion of HDR brachytherapy, patients received EBRT using a dynamic-arc conformal technique, administered with high energy photons comprising 10-MV X-rays to a total dose of 30 Gy. Total dose was administered in daily fraction of 3 Gy per fraction for 10 days over 2 weeks. The radiation field was limited to the prostate gland with or without proximal

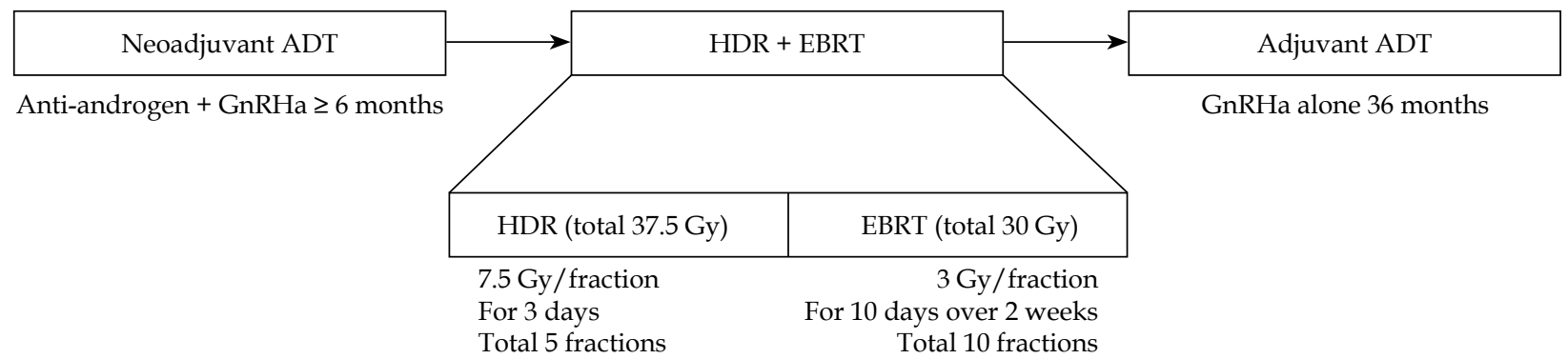

$A D T$ - androgen deprivation therapy, HDR - high-dose-rate brachytherapy, EBRT - external beam radiation therapy, GnRHa - gonadotropin-releasing hormone agonist injection

Fig. 1. Treatment protocol for high-dose-rate brachytherapy, external beam radiation therapy and hormonal therapy in highrisk and locally advanced prostate cancer 
seminal vesicles with a $7 \mathrm{~mm}$ leaf margin using multileaf collimators. No dose limitations for organs at risk were set in EBRT planning. We did not deliver pelvic irradiation beyond the prostate gland and seminal vesicles. On the assumption, $\alpha$ : $\beta$ ratio was 2 and 3 Gy for prostate and for organs (urethra/rectum) at risk, respectively. Biological effective dose of this combination therapy was $206 \mathrm{~Gy}$ for prostate and $158 \mathrm{~Gy}$ for organs at risk, respectively. Equivalent dose in 2 Gy fractions was 103 Gy for prostate and $95 \mathrm{~Gy}$ for organs at risk, respectively.

All patients initially underwent at least 6 months of neoadjuvant ADT, and adjuvant ADT was continued for 36 months after completion of radiotherapy. Neoadjuvant ADT combined nonsteroidal anti-androgen agents with gonadotropin-releasing hormone agonist (GnRHa) injections. Adjuvant ADT consisted of injections of GnRHa alone. Either flutamide $(375 \mathrm{mg} / \mathrm{d})$ or bicalutamide $(80 \mathrm{mg} / \mathrm{d})$ were used as the nonsteroidal anti-androgen agents. Either goserelin $(3.6 \mathrm{mg} / \mathrm{mo}$ or $10.8 \mathrm{mg} / 3 \mathrm{mo})$ or leuprorelin $(3.75 \mathrm{mg} / \mathrm{mo}$ or $11.25 \mathrm{mg} / 3 \mathrm{mo})$ were administrated as GnRHa therapy.

\section{Follow-up}

The duration of follow-up was calculated from the start of HDR brachytherapy. Patients with less than a year follow-up period were excluded from this study. The median follow-up for all patients was 84 months (range: 12-126 months). A follow-up examination after the HDR brachytherapy was scheduled every 3 months for the first year, and then 3 to 6 months thereafter. Biochemical failure was defined according to the Phoenix definition. The biochemical no evidence of disease (bNED) rate was calculated for all living patients and reflected biochemical failures.

\section{Statistical analysis}

For the purpose of analysis, clinical T stage (T1c-3a vs. T3b-4), Gleason score ( $\leq 7$ vs. $\geq 8$ ), biopsy positive core rates ( $<67 \%$ vs. $\geq 67 \%$ ), and nPSA after radiotherapy $(\leq 0.02 \mathrm{ng} / \mathrm{ml}$ vs. $>0.02 \mathrm{ng} / \mathrm{ml})$ were evaluated as dichotomous variables. Prostate-specific antigen nadir was defined as the lowest PSA measurement before any failure. In patients without failure, nPSA was the lowest PSA measurement during the entire follow-up period. The Wilcoxon rank-sum test and Student's $t$-test were used to evaluate for differences between post-radiotherapy nPSA groups for categorical variables and continuous variables, respectively. Univariate Cox regression models were used to examine the predictive value of patient related factors. Multivariate Cox regression models were created based on the covariates that were significant in univariate analysis. The Kaplan-Meier method was used to calculate survival functions. The AUC and best cutoff point of nPSA and time to achieve a PSA level of $\leq 0.02 \mathrm{ng} / \mathrm{ml}$ were calculated employing receiver operating characteristic (ROC) analysis. Differences were regarded as statistically significant at the $p<0.05$ level. Analyses were performed using SPSS, version 11.0 for Windows (SPSS, Inc., Chicago, IL, USA), GraphPad Prism, version 5 (GraphPad
Software, Inc., CA), and Microsoft Excel (Microsoft, Redmond, WA, USA).

\section{Results}

Of the 223 patients, 4 failed to complete the scheduled protocol for radiotherapy because they wished to discontinue HDR brachytherapy before the completion of the scheduled protocol. Additionally, three patients with less than a year follow-up period were excluded from this study. Analyses were thus performed for 216 patients. Of the 216 patients, 14 patients did not complete the adjuvant ADT because of loss to follow-up $(n=6,2.7 \%)$ and serious diseases $(n=8,3.7 \%)$. All patients reached their levels of nPSA before the completion of adjuvant ADT. One hundred eighty-eight patients $(87 \%)$ measured their levels of serum testosterone during the adjuvant ADT. Of those 188 patients, their levels of serum testosterone were at the castrate testosterone levels when patients reached their levels of nPSA.

The highest Radiation Therapy Oncology Group (RTOG)-defined acute genitourinary (GU) toxicities were grade 2 in 32 patients $(14.8 \%)$ and grade 3 in 10 patients $(4.6 \%)$. No patients experienced $\geq$ grade 2 acute gastrointestinal (GI) toxicities. The highest RTOG-defined late GU toxicities were grade 2 in 21 patients $(9.7 \%)$ and grade 3 in 21 patients $(9.7 \%)$. The most grade $3 \mathrm{GU}$ toxicities were urinary retention or urethral stricture, which were managed successfully by temporary catheterization or internal urethrotomy. The highest late GI toxicities were grade 2 in 5 patients $(2.8 \%)$. No patients showed grade 3 late GI toxicities. No patients experienced grade 4 or 5 toxicities.

The 7-year bNED rate, cancer specific-survival rate, and overall survival rate were $87.8 \%, 97.9 \%$, and $95.2 \%$, respectively. Twenty-two patients experienced biochemical failure. Of those 22 patients, the site of recurrence in 13 patients included bone metastasis $(n=7)$, distant lymph-node metastasis $(n=2)$, regional lymph-node metastasis $(n=1)$, lung metastasis $(n=1)$, and local recurrence $(n=2)$. Eleven patients died during follow-up, with causes including interstitial pneumonitis $(n=1)$, lung cancer $(n=1)$, subarachnoid hemorrhage $(n=1)$, cardiac disease $(n=1)$, an accident $(n=1)$, bladder cancer $(n=2)$, and prostate cancer $(n=4)$.

Characteristics of 216 patients are shown in Table 1. ROC curve was performed to evaluate an optimal cutpoint of nPSA after radiotherapy as a predictive value for biochemical failure (Figure 2). With a cutoff point of $0.02 \mathrm{ng} / \mathrm{ml}$, the sensitivity and specificity were $59.0 \%$ and $96.9 \%$, respectively. The AUC-ROC was 0.815 (95\% CI: $0.697-0.933, p<0.001)$. Using this cut-off point, we compared clinical and pathological features between a group with nPSA after radiotherapy of $\leq 0.02 \mathrm{ng} / \mathrm{ml}$ and that with $>0.02 \mathrm{ng} / \mathrm{ml}$. The clinical stage and biopsy positive core rate were significantly higher in a group with nPSA of $>0.02 \mathrm{ng} / \mathrm{ml}$ after radiotherapy than that with $\leq 0.02 \mathrm{ng} / \mathrm{ml}$ ( $p=0.040$ and 0.036 , respectively). There were no differences between the 2 groups with respect to other characteristics, including age, initial PSA, duration of neoadjuvant ADT, follow-up period, and proportion of Gleason score. 
Table 1. Comparison of clinical and pathological features between a group with PSA nadir after radiotherapy of $\leq 0.02 \mathrm{ng} / \mathrm{ml}$ and that with $>0.02 \mathrm{ng} / \mathrm{ml}$

\begin{tabular}{|c|c|c|c|c|}
\hline \multirow[t]{2}{*}{ Factors } & Total $(n=216)$ & $\mathrm{nPSA} \leq 0.02(n=196)$ & nPSA $>0.02(n=20)$ & \multirow[t]{2}{*}{$p$} \\
\hline & Median (range) & Median (range) & Median (range) & \\
\hline Age, years & $70(53-86)$ & $70(53-86)$ & $69(54-77)$ & 0.080 \\
\hline Initial PSA, ng/ml & $24(3-338)$ & $23(3-337)$ & $28(5-167)$ & 0.288 \\
\hline Neoadjuvant ADT, months & $12(4-74)$ & $12(4-74)$ & $12(7-17)$ & 0.358 \\
\hline \multirow[t]{2}{*}{ Follow-up, months } & $84(12-126)$ & $84(12-126)$ & $81(13-119)$ & 0.271 \\
\hline & $n(\%)$ & $n(\%)$ & $n(\%)$ & \\
\hline Gleason score & & & & 0.950 \\
\hline$\leq 6$ & 24 (11) & $21(11)$ & $3(15)$ & \\
\hline 7 & $102(47)$ & $94(48)$ & $8(40)$ & \\
\hline $8-10$ & $90(42)$ & $81(41)$ & $9(45)$ & \\
\hline T stage (2009) & & & & 0.040 \\
\hline $1 c-2 a$ & $55(26)$ & $51(26)$ & $4(20)$ & \\
\hline $2 b-2 c$ & $48(22)$ & $45(23)$ & $3(15)$ & \\
\hline $3 a$ & $75(35)$ & $71(36)$ & $4(20)$ & \\
\hline $3 b$ & $35(16)$ & $28(14)$ & $7(35)$ & \\
\hline 4 & $3(1)$ & $1(1)$ & $2(10)$ & \\
\hline Biopsy positive core rate & & & & 0.036 \\
\hline$<34 \%$ & $71(32)$ & $68(35)$ & $3(15)$ & \\
\hline $34-67 \%$ & $77(36)$ & $70(36)$ & $7(35)$ & \\
\hline$>67 \%$ & $65(30)$ & $55(27)$ & $10(50)$ & \\
\hline Unknown & $3(2)$ & $3(2)$ & $0(0)$ & \\
\hline
\end{tabular}

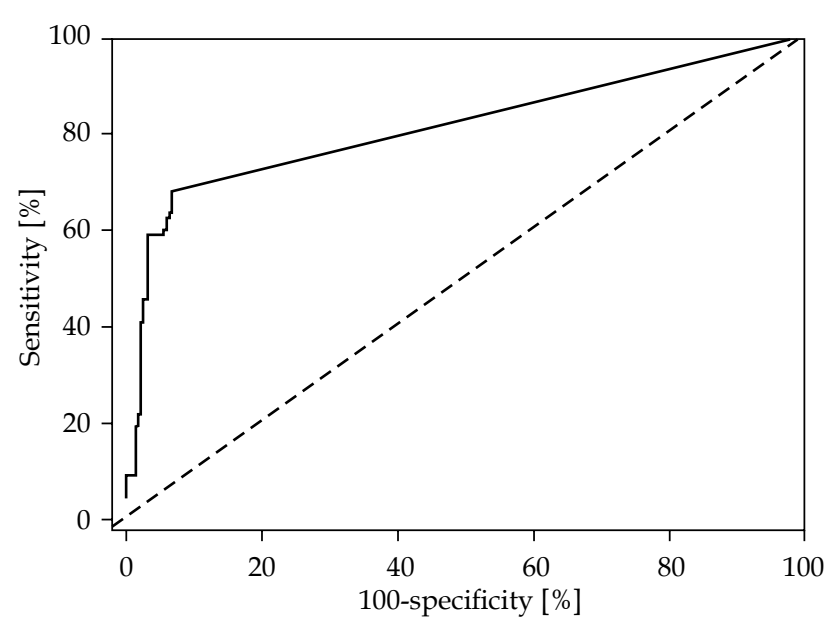

Fig. 2. Receiver operating characteristic (ROC) curve analysis to evaluate an optimal cut-point of prostate specific antigen nadir after the radiotherapy as a predictive value for biochemical failure (AUC-ROC $=0.815,95 \%$ CI: 0.697 $0.933, p<0.001)$
Table 2 summarizes pre-radiotherapy predictive values for worse biochemical control. Univariate analysis indicated that higher level of initial PSA (hazard ratio $[\mathrm{HR}]=1.006$; 95\% CI: 1.002-1.011; $p=0.008)$, Gleason score $\geq 8$ (HR $=3.164 ; 95 \%$ CI: 1.290-7.751; $p=0.011)$, biopsy positive core rate $\geq 67 \%(\mathrm{HR}=4.464 ; 95 \% \mathrm{CI}$ : $1.872-10.63$; $p=0.007)$, and T3b-T4 (HR = 3.623; 95\% CI: $1.550-8.474$; $p=0.003$ ) were significantly associated with increased risk of biochemical failure. An nPSA of $>0.02 \mathrm{ng} / \mathrm{ml}$ after radiotherapy was significantly associated with increased risk of biochemical failure on univariate analysis $(\mathrm{HR}=28.57$; 95\% CI: 12.04-66.66; $p<0.001)$. The 7-year bNED rate was $22.9 \%$ and $94.1 \%$ in patients with nPSA after radiotherapy $>0.02 \mathrm{ng} / \mathrm{ml}$ and $\leq 0.02 \mathrm{ng} / \mathrm{ml}$, respectively (Figure 3A).

Upon multivariate analysis, the nPSA value after radiotherapy was a significant independent predictor of biochemical failure ( $\mathrm{HR}=26.31 ; 95 \% \mathrm{CI}$ : 10.00-71.42; $p<0.001)$, whereas pre-radiotherapy predictive values for worse biochemical control-including higher level of initial PSA, Gleason score $\geq 8$, positive biopsy core rate $\geq 67 \%$, and T3b-T4-failed to reach independent predictor status (Table 2). 
Table 2. Predictive factors for worse biochemical control on proportional hazard model

\begin{tabular}{|c|c|c|c|c|c|c|c|c|}
\hline \multirow[t]{2}{*}{ Factors } & \multirow[t]{2}{*}{$N$ (event) } & \multicolumn{3}{|c|}{ Univariate } & \multirow{2}{*}{$\begin{array}{l}\text { bNED at } \\
7 \text { year, \% }\end{array}$} & \multicolumn{3}{|c|}{ Multivariate } \\
\hline & & $\mathrm{HR}$ & $95 \% \mathrm{Cl}$ & $p$ & & HR & $95 \% \mathrm{Cl}$ & $p$ \\
\hline Age & $216(22)$ & 0.944 & $0.880-1.013$ & 0.108 & & & & \\
\hline Duration of neoadjuvant ADT & $216(22)$ & 0.987 & $0.909-1.071$ & 0.746 & & & & \\
\hline Initial PSA & $216(22)$ & 1.006 & $1.002-1.011$ & 0.008 & & 1.003 & $0.995-1.012$ & 0.483 \\
\hline Gleason score & & & & 0.011 & & & & 0.068 \\
\hline$<8$ & $126(7)$ & 1.000 & (reference) & & 93.7 & 1.000 & (reference) & \\
\hline$\geq 8$ & $90(15)$ & 3.164 & $1.290-7.751$ & & 79.1 & 2.551 & $0.931-6.993$ & \\
\hline Biopsy positive core rates & & & & 0.007 & & & & 0.141 \\
\hline$<67 \%$ & $148(8)$ & 1.000 & (reference) & & 93.7 & 1.000 & (reference) & \\
\hline$\geq 67 \%$ & $65(14)$ & 4.464 & $1.872-10.63$ & & 72.9 & 2.336 & $0.753-7.246$ & \\
\hline T stage (2009 AJCC) & & & & 0.003 & & & & 0.605 \\
\hline T1c-Т3a & $178(13)$ & 1.000 & (reference) & & 91.2 & 1.000 & (reference) & \\
\hline T3b-T4 & $38(9)$ & 3.623 & $1.550-8.474$ & & 71.4 & 0.759 & $0.266-2.164$ & \\
\hline PSA nadir after radiotherapy & & & & $<0.001$ & & & & $<0.001$ \\
\hline$\leq 0.02 \mathrm{ng} / \mathrm{ml}$ & $20(9)$ & 1.000 & (reference) & & 94.1 & 1.000 & (reference) & \\
\hline$>0.02 \mathrm{ng} / \mathrm{ml}$ & 196 (13) & 28.57 & $12.04-66.66$ & & 22.9 & 26.31 & $10.00-71.42$ & \\
\hline
\end{tabular}

$H R$ - hazard ratio, Cl - confidence interval, bNED - biochemical no evidence of disease, ADT - androgen deprivation therapy, PSA - prostate specific antigen, AJCC - American Joint Committee on Cancer

Prostate cancer specific-survival rate was significantly lower in patients with nPSA $>0.02 \mathrm{ng} / \mathrm{ml}$ after radiotherapy than in patients with lower nPSA values $(\mathrm{HR}=32.25$; 95\% CI: 3.401-333.3; $p=0.002)$. The 7-year cancer specific-survival rate was $82.4 \%$ and $99.4 \%$ in patients with nPSA after radiotherapy $>0.02 \mathrm{ng} / \mathrm{ml}$ and $\leq 0.02 \mathrm{ng} / \mathrm{ml}$, respectively (Figure 3B).

ROC curve was performed to evaluate an optimal cutpoint of time to achieve a PSA level of $\leq 0.02 \mathrm{ng} / \mathrm{ml}$ after radiotherapy as a predictive value for biochemical failure. With a cutoff point of 1 year, the sensitivity and specificity were $89.9 \%$ and $60 \%$, respectively. The AUC-ROC was 0.815 (95\% CI: $0.639-0.944, p<0.001$ ). Of 216 patients in our cohort, 196 patients reached a PSA level of $\leq 0.02 \mathrm{ng} / \mathrm{ml}$ during the entire follow-up period. Using this cut-off point, 196 patients who reached a PSA level of $\leq 0.02 \mathrm{ng} / \mathrm{ml}$ were divided into 2 groups according to how long it took to achieve that level: $\leq 1$ year after radiotherapy $(n=171)$ and $>1$ year after radiotherapy $(n=25)$. Biochemical failure rate was significantly higher in patients with PSA level of $\leq 0.02$ achieved $>1$ year after radiotherapy than in those with $\leq 1$ year (HR $=15.38$; 95\% CI: 3.846-62.50; $p<0.001)$. The 7 -year bNED rate was $70.8 \%$ and $97.5 \%$ in patients with PSA level of $\leq 0.02$ achieved $>1$ year after radiotherapy and in those with $\leq 1$ year, respectively (Figure 4 ).

\section{Discussion}

This report restricted evaluation to high-risk and locally advanced prostate cancer, and analyzed the prognostic value of nPSA after combined HDR brachytherapy and hypofractionated EBRT in patients treated with longterm ADT with a median follow-up of 7 years. A nPSA of $>0.02 \mathrm{ng} / \mathrm{ml}$ after radiotherapy was a significant independent predictor of biochemical failure, whereas none of the pretreatment predictive values reached indepen- dent predictor status - including higher level of initial PSA, Gleason score $\geq 8$, positive biopsy core rate $\geq 67 \%$, and T3b-T4. Patients whose PSA levels did not decline to $\leq 0.02 \mathrm{ng} / \mathrm{ml}$ after radiotherapy had a high possibility of prostate cancer-related death.

Previous studies suggested that nPSA after prostate radiotherapy is well correlated with prognostic values, including prostate cancer specific mortality. Threshold levels of nPSA for the prediction of treatment failure vary depending on the local extent of the cancer, the type of radiotherapy, and the duration of ADT. Our value of nPSA for predicting the clinical outcome is an extremely low level compared with previous studies - ranging from 0.1 to $1.5 \mathrm{ng} / \mathrm{ml}[17,18,19,20]$. Combined HDR brachytherapy and EBRT could deliver effective radiation doses more precisely and intensely than conventional EBRT, with some margin even in patients with extracapsular extensions and seminal vesicle invasion. Precise local control induced by this dose intensification could be one reason for the lower level of nPSA threshold in our study compared with previous studies. In addition, Japanese-specific high sensitivity to hormonal therapy may have a role in the lower nPSA values [21]. In our regimen, all patients initially underwent $\geq 6$ months of neoadjuvant ADT (median 12 months). This adequate period of neoadjuvant ADT may have also affected the lower nPSA value in our study.

Patients whose PSA levels have not reached favorable thresholds may require careful observation and additional treatment because they potentially have a greater risk for prostate-cancer specific death. We hypothesize that a PSA level that does not reach $\leq 0.02 \mathrm{ng} / \mathrm{ml}$ after radiotherapy may reflect occult distant metastasis present at the initial diagnosis. We did not deliver EBRT to pelvic lymph nodes where microscopic metastasis may be present. Patients with higher PSA levels, higher pathology 

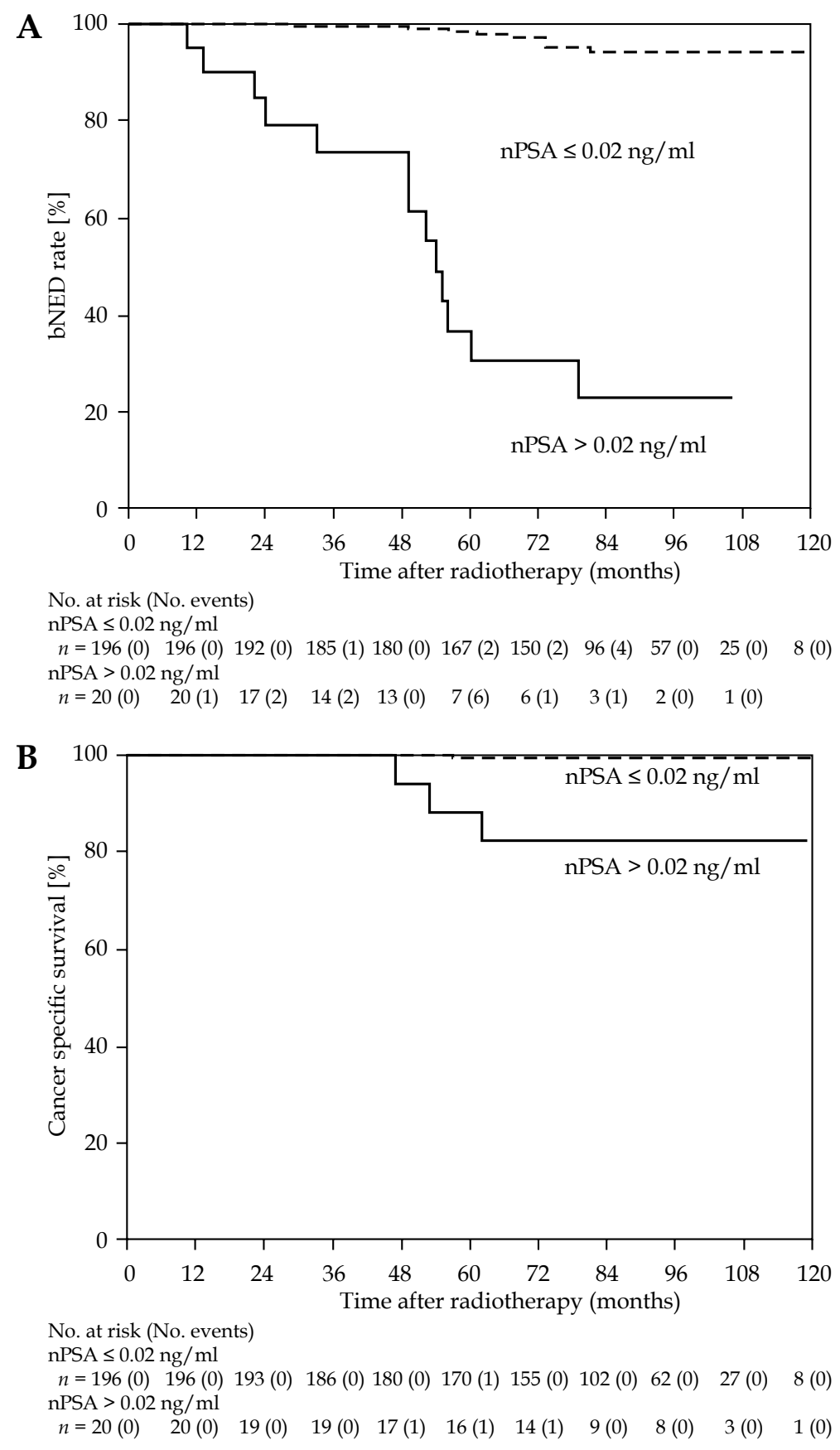

PSA - prostate-specific antigen, nPSA - PSA nadir after radiotherapy, bNED - biochemical no evidence of disease

Fig. 3. Kaplan-Meier estimate of (A) biochemical no evidence of disease rate and (B) prostate cancer specific-survival rate based on prostate-specific antigen nadir (nPSA) after high-dose-rate brachytherapy combined with external beam radiation therapy, respectively. Patients were divided into 2 groups according to levels of nPSA after radiotherapy: nPSA of $\leq 0.02 \mathrm{ng} / \mathrm{ml}(n=196)$ and nPSA of $>0.02 \mathrm{ng} / \mathrm{ml}(n=20)$

grade, and a higher percentage of positive biopsy cores at initial diagnosis are more likely to have lymph node involvement than those with lower values $[22,23]$. Additional irradiation into the pelvis where lymph node metastasis may be present has the potential to improve the outcome in patients whose PSA levels have not reached favorable thresholds. However, in the context of locally advanced prostate cancer it remains controversial whether extended radiotherapy such as whole-pelvic radiother- apy yields improved survival compared to prostate and seminal vesicle radiotherapy, especially in patients who are also treated with ADT [24,25,26,27].

Of 196 patients who reached a PSA level of $\leq 0.02 \mathrm{ng} /$ $\mathrm{ml}$, biochemical failure rate was significantly higher in patients who reached that level achieved $>1$ year after radiotherapy than in those with $\leq 1$ year (Figure 4 ). Not only lower levels of PSA decline but also earlier decline of low levels of PSA after completing radiotherapy are predic- 


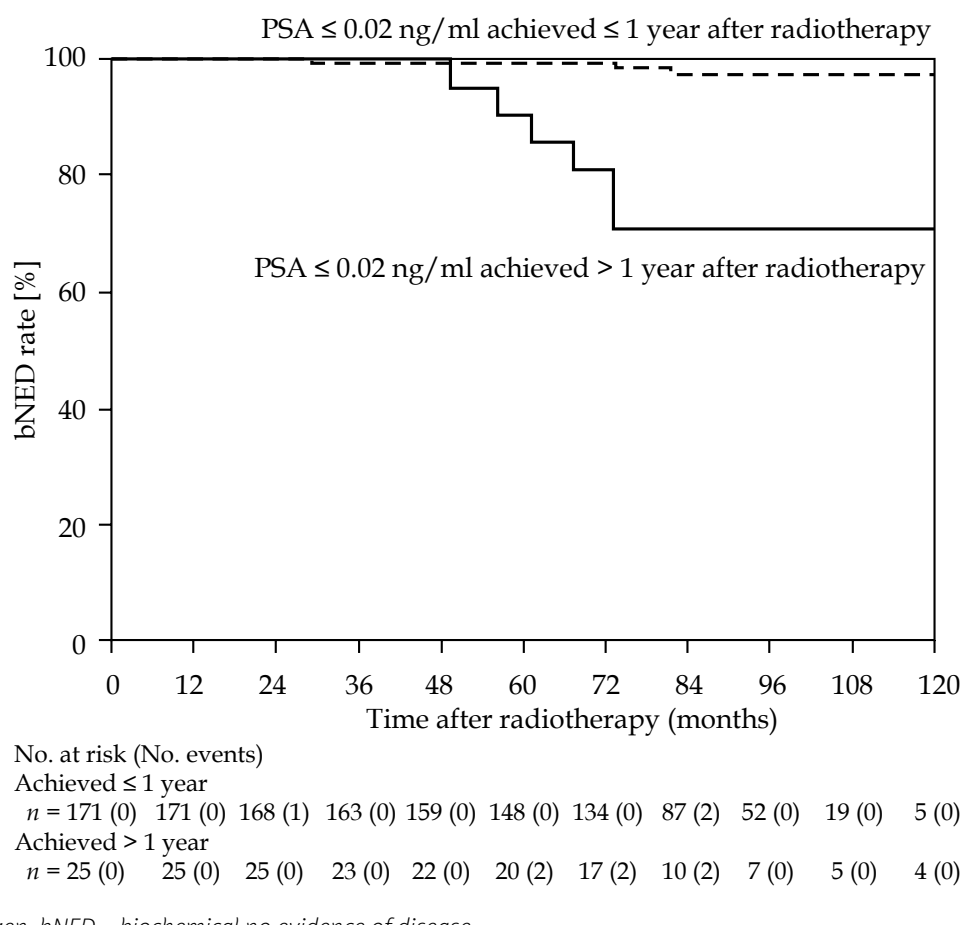

PSA - prostate-specific antigen, bNED - biochemical no evidence of disease

Fig. 4. Kaplan-Meier estimate of biochemical no evidence of disease rate in 196 patients who reached a prostate-specific antigen (PSA) level of $\leq 0.02 \mathrm{ng} / \mathrm{ml}$. Patients were divided into 2 groups according to how long it took to achieve that level: $\leq 1$ year after radiotherapy $(n=171)$ and $>1$ year after radiotherapy $(n=25)$

tive for long-term biochemical relapse-free survival. Our data suggest that the lowest PSA level achieved during the first year after completing radiotherapy may be an early surrogate marker for long-term biochemical control. Cavanaugh et al. [28] suggested the prognostic value of a time-and-PSA threshold model following prostate carcinoma radiotherapy without ADT. They analyzed the prognostic value of early PSA kinetics, with PSA assessed as reaching or failing to reach discrete threshold values at fixed time points after EBRT. Significantly improved bNED were found in a comparison of patients whose PSA levels reached favorable thresholds and patients whose PSA levels did not reach those thresholds at early fixed time points ( 3 and 6 months of follow-up). Alcántara et al. [29] suggested that the nPSA level at 1 year is a strong independent predictor of outcome after EBRT in patients treated without ADT. Dichotomized nPSA at 1 year ( $\leq 2 \mathrm{vs} .>2 \mathrm{ng} / \mathrm{ml}$ ) was independently related to distant metastasis and cause-specific mortality. Some studies have suggested that PSA response to neoadjuvant ADT before EBRT predicts long-term prostate cancer survival outcome [30,31]. Long-term follow-up is usually required to evaluate the treatment efficacy in prostate cancer. If our nPSA value could predict clinical recurrence during an early phase of treatment, timely salvage treatment could be initiated in patients with a poor prognosis, potentially preventing prostate cancer-related death. The ability to identify patients at increased risk of death from prostate cancer in early phases of treatment helps to promote demand for alternative treatments including new clinical trials and more aggressive therapies [28,31,32]. Addi- tional aggressive therapies are required to salvage patients who did not reach the favorable PSA level after the prostate radiotherapy. However, there exists no effective treatment modality to salvage those patients at present.

Several potential limitations of this study must be considered in addition to its retrospective nature. Although patients in this study received at least 6 months of neoadjuvant ADT, the duration of neoadjuvant ADT was not uniform. The sensitivity of hormonal therapy and the type of nonsteroidal anti-androgen agents were different in each patient. Those factors may affect the analysis for prognostic value of nPSA. We did not eliminate those effects of hormonal therapy on the prognostic value of nPSA in the ROC analysis to find an optimal cut-point of nPSA after radiotherapy. In addition, the use of EBRT in combination with HDR brachytherapy may also affect the analysis for the value of the nPSA. Second, prolonged sustainment of castration testosterone levels after cessation of long-term ADT occurred in approximately $20 \%$ of patients in our cohort (data not shown) [33]. The prolonged sustainment of the testosterone levels could affect the biochemical control rates. Third, the choice of patient population might not be the best choice to correlate the value of the nPSA to biochemical failure because the clinical stage and biopsy positive core rate were significantly higher in a group with nPSA of $>0.02 \mathrm{ng} / \mathrm{ml}$ after radiotherapy than that with $\leq 0.02 \mathrm{ng} / \mathrm{ml}$. Fourth, our median follow-up of 84 months may be insufficient to address the clinical outcome of non-metastatic prostate cancer. Further follow-up will be needed to address whether our lower PSA threshold could be predictive for overall and 
cause-specific survival over an extended time period. Results from longer follow-up survival data will clarify the utility of our PSA threshold model for predicting the clinical outcome.

\section{Conclusions}

For patients with locally advanced and clinically non-metastatic high-risk prostate cancer who are treated with a combination of HDR brachytherapy, hypofractionated EBRT, and long-term ADT, our data demonstrate that a post-radiotherapy nPSA value of $\leq 0.02 \mathrm{ng} /$ $\mathrm{ml}$ is associated with better long-term biochemical tumor control and cause-specific survival, even if patients had pretreatment predictive values for worse control. In addition, patients who reached PSA levels of $\leq 0.02 \mathrm{ng} / \mathrm{ml}$ within 1 year after radiotherapy had significantly improved biochemical tumor control. We have to consider more aggressive treatment in patients whose PSA levels have not reached a favorable threshold because they have potential risks for death from prostate cancer.

\section{Disclosure}

Dr Tsumura received honoraria for lecture fees from Takeda Pharmaceutical Co, Ltd. The other authors report no conflict of interest.

\section{References}

1. Yoshioka Y, Suzuki O, Otani Y et al. High-dose-rate brachytherapy as monotherapy for prostate cancer: technique, rationale and perspective. J Contemp Brachytherapy 2014; 6: 91-98.

2. Morton GC. High-dose-rate brachytherapy boost for prostate cancer: rationale and technique. J Contemp Brachytherapy 2014; 6: 323-330

3. Cendales R, Alwers E, Cifuentes J et al. High-dose-rate brachytherapy delivered in two fractions as monotherapy for lowrisk prostate cancer. J Contemp Brachytherapy 2015; 7: 10-16.

4. Hoskin PJ, Motohashi K, Bownes P et al. High dose rate brachytherapy in combination with external beam radiotherapy in the radical treatment of prostate cancer: initial results of a randomized phase three trial. Radiother Oncol 2007; 84: 114-120.

5. Ishiyama H, Kitano M, Satoh $\mathrm{T}$ et al. Genitourinary toxicity after high-dose-rate (HDR) brachytherapy combined with Hypofractionated External beam radiotherapy for localized prostate cancer: an analysis to determine the correlation between dose-volume histogram parameters in HDR brachytherapy and severity of toxicity. Int J Radiat Oncol Biol Phys 2009; 75: 23-28.

6. Challapalli A, Jones E, Harvey $\mathrm{C}$ et al. High dose rate prostate brachytherapy: an overview of the rationale, experience and emerging applications in the treatment of prostate cancer. Br J Radiol 2012; 85 Spec No 1: S18-S27.

7. Ishiyama $H$, Satoh $\mathrm{T}$, Kitano $\mathrm{M}$ et al. High-dose-rate brachytherapy and hypofractionated external beam radiotherapy combined with long-term hormonal therapy for high-risk and very high-risk prostate cancer: outcomes after 5-year follow-up. J Radiat Res 2014; 55: 509-517.

8. Brenner DJ. The linear-quadratic model is an appropriate methodology for determining isoeffective doses at large doses per fraction. Semin Radiat Oncol 2008; 18: 234-239.

9. Bolla M, Collette L, Blank L et al. Long-term results with immediate androgen suppression and external irradiation in patients with locally advanced prostate cancer (an EORTC study): a phase III randomized trial. Lancet 2002; 360: 103-106.

10. Horwitz EM, Bae K, Hanks GE et al. Ten-year follow-up of radiation therapy oncology group protocol 92-02: a phase III trial of the duration of elective androgen deprivation in locally advanced prostate cancer. J Clin Oncol 2008; 26: 2497-2504.

11. Zapatero A, Guerrero A, Maldonado X et al. High-dose radiotherapy with short-term or long-term androgen deprivation in localized prostate cancer (DART01/05 GICOR): a randomized, controlled, phase 3 trial. Lancet Oncol 2015; 16: 320-327.

12. Galalae RM, Martinez A, Mate T et al. Long-term outcome by risk factors using conformal high-dose-rate brachytherapy (HDR-BT) boost with or without neoadjuvant androgen suppression for localized prostate cancer. Int J Radiat Oncol Biol Phys 2004; 58: 1048-1055.

13. Kaprealian T, Weinberg V, Speight JL et al. High-dose-rate brachytherapy boost for prostate cancer: comparison of two different fractionation schemes. Int J Radiat Oncol Biol Phys 2012; 82: 222-227.

14. Khor R, Duchesne G, Tai KH et al. Direct 2-arm comparison shows benefit of high-dose-rate brachytherapy boost vs external beam radiation therapy alone for prostate cancer. Int J Radiat Oncol Biol Phys 2013; 85: 679-685.

15. Kamrava M, Rwigema JC, Chung Met al. Predictors of distant metastasis after combined HDR brachytherapy and external beam radiation for prostate cancer. J Contemp Brachytherapy 2013; 5: 127-133.

16. D'Amico AV, Whittington R, Malkowicz SB et al. Biochemical outcome after radical prostatectomy, external beam radiation therapy, or interstitial radiation therapy for clinically localized prostate cancer. JAMA 1998; 280: 969-974.

17. Grimm PD, Blasko JC, Sylvester JE et al. 10-year biochemical (prostate-specific antigen) control of prostate cancer with (125) I brachytherapy. Int J Radiat Oncol Biol Phys 2001; 51: 31-40.

18. Ray ME, Thames HD, Levy LB et al. PSA nadir predicts biochemical and distant failures after external beam radiotherapy for prostate cancer: a multi-institutional analysis. Int J Radiat Oncol Biol Phys 2006; 64: 1140-1150.

19. Zelefsky MJ, Shi W, Yamada Y et al. Postradiotherapy 2-year prostate-specific antigen nadir as a predictor of long-term prostate cancer mortality. Int J Radiat Oncol Biol Phys 2009; 75: 1350-1356.

20. Morris LM, Izard MA, Wan WY. Does prostate-specific antigen nadir predict longer-term outcomes of prostate cancer after neoadjuvant and adjuvant androgen deprivation therapy in conjunction with brachytherapy? Brachytherapy 2015; 14: 322-328

21. Fukagai T, Namiki TS, Carlile RG et al. Comparison of the clinical outcome after hormonal therapy for prostate cancer between Japanese and Caucasian men. BJU Int 2006; 97: 1190-1193.

22. Briganti A, Larcher A, Abdollah F et al. Updated nomogram predicting lymph node invasion in patients with prostate cancer undergoing extended pelvic lymph node dissection: the essential importance of percentage of positive cores. Eur Urol 2012; 61: 480-487.

23. Gacci M, Schiavina R, Lanciotti $M$ et al. External validation of the updated nomogram predicting lymph node invasion in patients with prostate cancer undergoing extended pelvic lymph node dissection. Urol Int 2013; 90: 277-282.

24. Roach M 3rd, DeSilvio M, Lawton C et al. Phase III trial comparing whole-pelvic versus prostate-only radiotherapy and neoadjuvant versus adjuvant combined androgen suppression: Radiation Therapy Oncology Group 9413. J Clin Oncol 2003; 21: 1904-1911.

25. Pommier P, Chabaud S, Lagrange JL et al. Is there a role for pelvic irradiation in localized prostate adenocarcinoma? Preliminary results of GETUG-01. J Clin Oncol 2007; 25: 5366-5373. 
26. Aizer AA, Yu JB, McKeon AM et al. Whole pelvic radiotherapy versus prostate only radiotherapy in the management of locally advanced or aggressive prostate adenocarcinoma. Int J Radiat Oncol Biol Phys 2009; 75: 1344-1349.

27. Bittner N, Merrick GS, Wallner KE et al. Whole-pelvis radiotherapy in combination with interstitial brachytherapy: does coverage of the pelvic lymph nodes improve treatment outcome in high-risk prostate cancer? Int J Radiat Oncol Biol Phys 2010; 76: 1078-1084.

28. Cavanaugh SX, Kupelian PA, Fuller CD et al. Early prostate-specific antigen (PSA) kinetics following prostate carcinoma radiotherapy: prognostic value of a time-and-PSA threshold model. Cancer 2004; 101: 96-105.

29. Alcántara P, Hanlon A, Buyyounouski MK et al. Prostatespecific antigen nadir within 12 months of prostate cancer radiotherapy predicts metastasis and death. Cancer 2007; 109: 41-47.

30. Zelefsky MJ, Gomez DR, Polkinghorn WR et al. Biochemical response to androgen deprivation therapy before external beam radiation therapy predicts long-term prostate cancer survival outcomes. Int J Radiat Oncol Biol Phys 2013; 86: 529-533.

31. McGuire SE, Lee AK, Cerne JZ et al. PSA response to neoadjuvant androgen deprivation therapy is a strong independent predictor of survival in high-risk prostate cancer in the dose-escalated radiation therapy era. Int J Radiat Oncol Biol Phys 2013; 85: e39-e46.

32. D'Amico AV, Moul JW, Carroll PR et al. Surrogate end point for prostate cancer-specific mortality after radical prostatectomy or radiation therapy. J Natl Cancer Inst 2003; 95: 1376-1383.

33. Tsumura $\mathrm{H}$, Satoh $\mathrm{T}$, Ishiyama $\mathrm{H}$ et al. Recovery of serum testosterone following neoadjuvant and adjuvant androgen deprivation therapy in men treated with prostate brachytherapy. World J Radiol 2015; 7: 494-500. 